\title{
Management of Uncertainty and Spatio-Temporal Aspects for Monitoring and Diagnosis in a Smart Home
}

\author{
Juan Carlos Augusto ${ }^{1}$ Jun Liu ${ }^{1}$ Paul McCullagh ${ }^{1}$ Hui Wang ${ }^{1}$ Jian-Bo Yang ${ }^{2}$ \\ ${ }^{1}$ School of Computing and Mathematics, University of Ulster at Jordanstown, \\ Newtownabbey BT37 OQB, Co. Antrim, UK \\ E-mail: [jc.augusto, j.liu,pj.mccullagh,h.wang]@ulster.ac.uk \\ ${ }^{2}$ Manchester Business School (East) \\ The University of Manchester \\ Manchester M15 6PB, UK \\ E-mail: jian-bo.yang@mbs.ac.uk \\ Received: 12-11-2008 \\ Revised: 13-11-2008
}

\begin{abstract}
The health system in developed countries is facing a problem of scalability in order to accommodate the increased proportion of the elderly population. Scarce resources cannot be sustained unless innovative technology is considered to provide health care in a more effective way. The Smart Home provides preventive and assistive technology to vulnerable sectors of the population. Much research and development has been focused on the technological side (e.g., sensors and networks) but less effort has been invested in the capability of the Smart Home to intelligently monitor situations of interest and act in the best interest of the occupants. In this article we model a Smart Home scenario, using knowledge in the form of Event-Condition-Action rules together with a new inference scheme which incorporates spatio-temporal reasoning and uncertainty. A reasoning system called RIMER, has been extended to permit the monitoring of situations according to the place where they occur and the specific order and duration of the activities. The system allows for the specification of uncertainty both in terms of knowledge representation and credibility of the conclusions that can be achieved in terms of the evidence available.
\end{abstract}

Keywords: Decision support, monitoring and diagnosis, ambient intelligence, Smart Home, uncertainty, spatio-temporal reasoning.

\section{Introduction}

The relative increased proportion of the elderly population due to demographic progression combined with advances in medical therapy means that people can live longer and health care at home is now a feasible and attractive area of application. There is also a growing tendency to decentralize health care, shifting from the hospital to the community and hence home-centred health care has become an important health management issue ${ }^{1,2}$. As a consequence of the ageing process the propensity to suffer chronic illnesses which demand close monitoring increases. Fortunately, advances in technology and problem solving skills are making available new options for assistive health care. Current research blends work in Ambient Intelligence with tasks related to monitoring, problem identification 
and emergency intervention. By Ambient Intelligence (AmI) ${ }^{3,4,5,6}$ here we mean "a digital environment that proactively, but sensibly, supports people in their daily lives" 7 . Other terms such as Ubiquitous Computing ${ }^{8}$ or Smart Environments ${ }^{9}$ are used with similar connotations.

A variety of environments can be used for the deployment of ambient intelligence. Typical places researchers are currently looking at include classrooms, cars, houses, offices, ambulances, hospitals, and airports ${ }^{3,10}$. The most well-known realization of the concept of Ambient Intelligence nowadays are Smart Homes (SH). By Smart Home here we mean "a house equipped with sensors and actuators which can be coordinated by intelligent software to benefit its inhabitants" 11. A Smart Home can help people at risk in their living place by preventing hazards and by assisting them as much as possible when they need health services 12,13,14. For example, people with dementia or Alzheimer's disease can live an independent life for longer under the protection of a $\mathrm{SH}^{15}$. Technology has made significant advances in developing sensors and networks that allow the monitoring of the environment, and the provision of alerts to users. However there has been insufficient progress on data analysis and interpretation to take full advantage of these technologies.

Intelligent monitoring is required to fully exploit the potential of this supported environment and some relevant studies have been reported. Williams et al. ${ }^{16}$ introduced intelligence into CarerNet, which simulated a SH to provide a telecare solution for a patient discharged from hospital. Sixsmith ${ }^{17}$ undertook a three month patient trial which identified unsafe conditions by detecting deviations from normal activity patterns. Twenty-two residents, aged over 60 years, generated 61 alerts (46 of which were false alarms, with 15 genuine alerts). Whilst no primary emergencies were encountered, the study determined that the supported environment enhanced feelings of safety and security, and stimulated independence of the residents and their carers.

An appreciation of spatio-temporal events and the lack of certainty in the temporal nature of these events are key to successfully monitoring dynamic activities within a SH. The importance of probabilis- tic reasoning in the area of clinical decision support was recognized by Shortliffe and utilized in the landmark rule based decision support system, MYCIN ${ }^{18}$. A theoretical treatment of constraints in temporal reasoning was investigated by Dechter et al. ${ }^{19}$, who developed a formalism that enabled representation of time constraints between activities. In a more recent clinical application of intelligent monitoring, Bellazzi et al. ${ }^{20}$ applied Bayesian estimation to investigate daily patterns of blood glucose level time series, from a diabetic patient. The problem was specified by stochastic equations, and solved using a Markov chain technique. In the context of the SH environment, Patterson et al. ${ }^{21}$ used a Radio Frequency Identification (RFID) enabled glove to monitor routine activities and study fine grained temporal activity, yielding context-aware information. They utilized probabilistic models for activity recognition, and investigated the impact of increasing complexity. With their approach they could reason about aggregated object instances and abstract to their classes, to provide a description of household activity.

In this article, we apply a methodology referred to as Rule-base Inference Methodology using the $E v$ idential Reasoning (RIMER) ${ }^{22}$, extended within an active database framework ${ }^{23}$ to investigate spatiotemporal aspects of human activities monitoring. Although there are many possible architectures with which to implement intelligent $\mathrm{SH}$ systems, rulebased systems offer a simple framework and are amenable to verification and validation ${ }^{24,4}$. Uncertainty is inevitable in a $\mathrm{SH}$ application due to the vagueness intrinsic to human communication, inaccuracy or incompleteness resulting from limited knowledge and the imprecision of instruments. It is therefore necessary to use a scheme for representing and processing vague, imprecise or incomplete information in conjunction with precise data.

The concept of a belief rule-base and its associated inference methodology were proposed in 22 as a formalism based on the Evidential Reasoning (ER) approach ${ }^{25,26,27}$. In a belief rule-base, each possible consequent of a rule is associated with a belief degree. Such a rule-base is capable of capturing complicated and even continuous causal re- 
lationship between different factors while the traditional IF-THEN rules are its special cases $28,29,30,31$.

Combining spatio-temporal reasoning with uncertainty reasoning captures essential concepts that we believe can improve the ways in which SHs can be designed. We address a case study in which the occupant has become motionless (possibly due to a fall or fainting) to test the knowledge representation and inference logic. A thorough practical community based assessment is beyond the scope of this work and has not been undertaken.

The article is structured as follows. Section 2 will provide a general description of SHs and the specific features of a SH scenario that will be used in the rest of the article. Section 3 will provide a background on what has been explored before in terms of monitoring and diagnosis in a SH system. The combination of space, time and uncertainty are explained in section 4 and exemplified in a $\mathrm{SH}$ setting in section 5. Conclusions and future work are outlined in section 6 .

\section{Smart Homes}

A Smart Home $32,33,34,35,36,37,38$ can be described as a house that is supplemented with technology, for example sensors and devices, in order to increase the range of services provided to its occupants by reacting in an intelligent way. The technology will have two main components: a set of sensors and a networking layer linking those sensors with computing facilities. Common sensors monitor carbon monoxide, smoke, heat, motion (as used for burglary alarms) and window or door opening. Some devices have been enriched with sensors to detect usage ${ }^{15}$. For example a sensor can detect that a water tap is open/closed or that a cooker is in use, or a microwave can scan a bar code on food to automate the cooking process. A layout plan of a $\mathrm{SH}$ enriched with sensors and devices is shown in Figure 1. This type of interface can be used to simulate activity or to display real world data. The SH comprises the following rooms/environment: reception, kitchen, toilet/bathroom, living room, bedroom, and the outside.

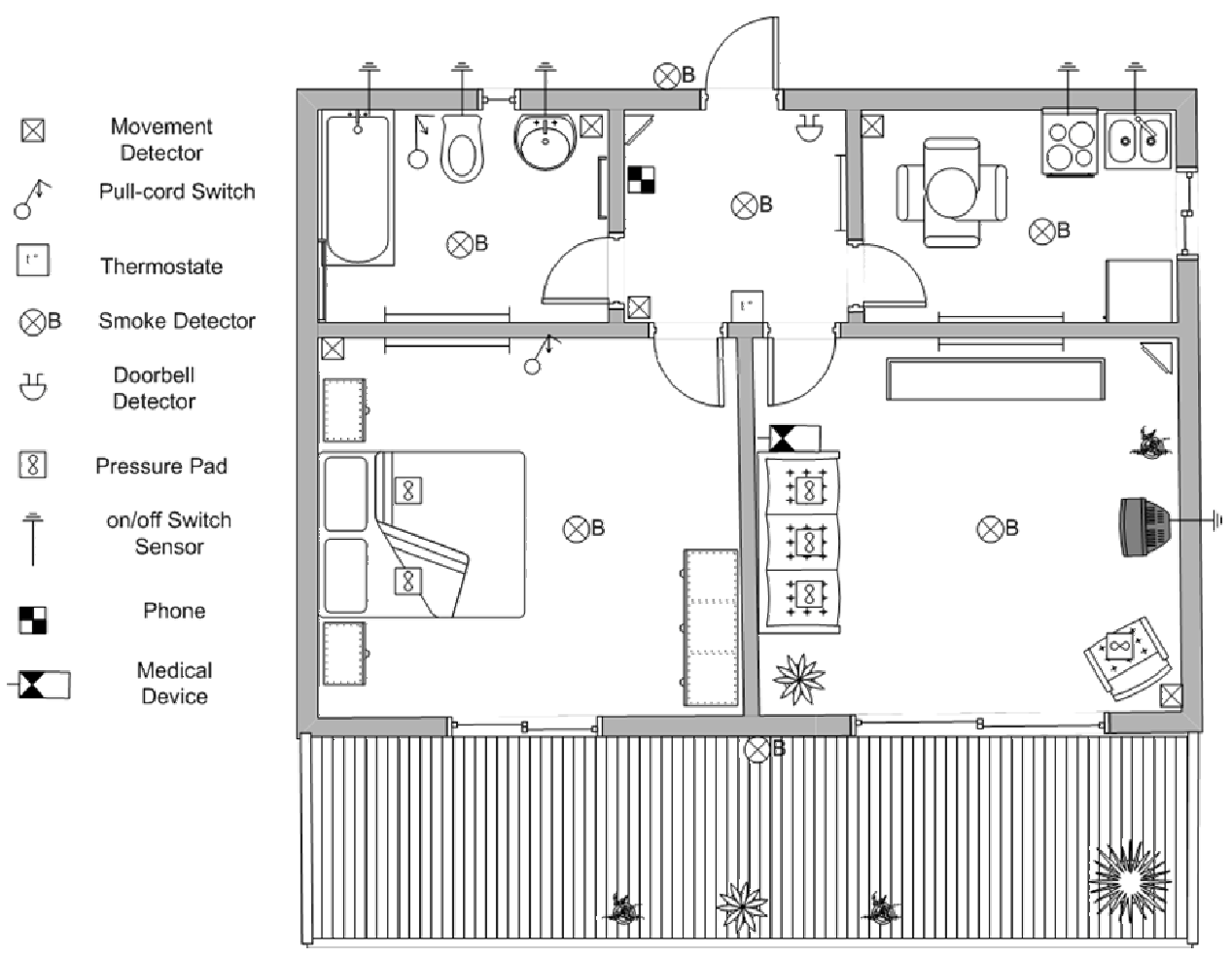

Fig. 1. The layout of an example Smart Home 
The network comprises a controller (computer) and wired or wireless infrastructure. The controller is normally located within the premises, but may be connected remotely via the Internet. It communicates with sensors so that for example, the cooker can be turned off automatically. An obvious way to turn off a device would be with a timer but this can be a very rigid mechanism. A more useful and flexible use of the device demands the intelligent analysis of several factors in order to decide if the turning off of a cooker is meaningful given a context. Many different devices can be used in a house to gain understanding of the activities of daily living (ADL) or at least to have more information of the context when particular situations of interest happen. This knowledge increases the possibilities of assessing situations and taking decisions correctly.

In order to illustrate our methodology we restrict ourselves to the use of basic sensors described in the previous paragraph and focus on the potential uses of this technology for health related monitoring. Detecting a transition in moving from room $\mathrm{A}$ to room $\mathrm{B}$ is represented by an event $t d A B_{-} \_n$. For example, $t d R K_{-}$on will represent that the person is activating an RFID sensor while passing through the door communicating the kitchen with the reception area. This event alone is not enough to detect the direction the occupant is moving to so disambiguation is needed with the help of the movement sensors. The activation of movement sensors in the kitchen is represented by at_kitchen_on and the absence of activation by at_kitchen_off. A similar convention is used with sensors at other rooms.

\section{An Investigation into Rule-Based Design of Smart Homes Systems}

\subsection{ECA rules}

Dynamic systems like Smart Homes can be modeled by considering the occurrence of meaningful events and the contexts in which those events occur to detect situations of interest, and enable decisions to be taken. Active databases ${ }^{39}$ can be used to store information gathered from a SH. A characteristic feature of Active Databases is their use of Event-ConditionAction (ECA) rules as a way to react to the incoming information. ECA rules have a syntax of the following format:

ON <Event>, IF <Condition>, DO <Action>

This means that whenever an occurrence of the event described in the ON clause is detected, if the condition described in the IF clause (usually imposing constraints on different aspects of the events described in the ON clause) is true, the action described in the DO clause is obeyed by the system. When the ON clause is satisfied the rule is said to be 'triggered' and if in addition to that the IF clause is satisfied then the rule is 'fired'.

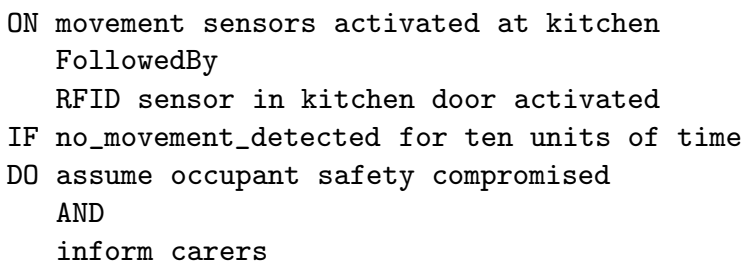

For example, the above rule is triggered when movement is detected in the kitchen, a spatial transition occurs and then no further movement in an adjoining room is detected. Under the conditions that the assisted occupant is known to be at home (this conclusion is the consequence of another rule or set of rules which can have as a resulting action: 'set status variable occupant_at_home=true') the action recommended is to initiate an intervention, e.g. request a visit from carer.

\subsubsection{Uncertainty in ECA rules}

Despite the growing research interest in SHs, relatively little work has been carried out in extending them to encompass the management of uncertain information. It is also generally accepted that whenever real world information is to be represented in a system, it will be of imperfect nature. Sources of uncertainty in ECA rules include:

- Uncertain event. The occurrence of the event described in the ON clause may be uncertain, e.g. "It is most likely that the occupant has fallen" or "The occupant is in the kitchen with $80 \%$ certainty".

- Uncertain condition. Uncertain conditions might include uncertain queries, e.g. "a sensor can be considered activated with 'high' confidence". 
- Uncertain relationship between the event/condition and the actions. Uncertainty may be caused by weak implication that may occur when an expert is unable to establish a precise correlation between the event/condition and the action except by using degrees of belief. One such situation may lead to the specification of a rule expressing that if some events are detected in a context suggesting a monitored person is active and they are followed (say 10 units of time later) by other events suggesting sudden suspension of activities, then there is a significant chance $(80 \%$ as determined by an expert) that the occupant may be in a compromised situation (e.g., has fallen or fainted). The antecedent event associated with the ON clause refers to continuously monitored events in the SH. In standard IF-THEN rules these events are often merged with the IF condition, and this terminology is adopted in the following examples.

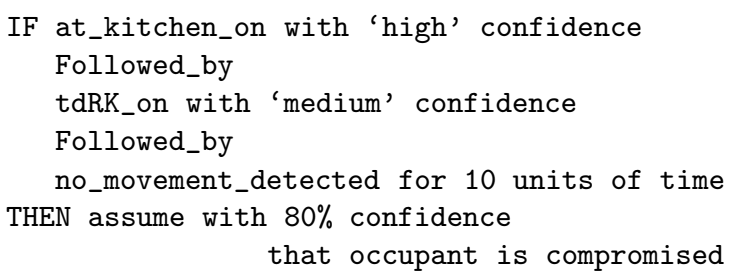

Depending on incoming sensor-related events, the system evaluates all the ECA-rules to identify which event part matches the actual situation. These selected rules may conflict with each other if the event parts of more than one rule are matched simultaneously. Resolving the conflict is a crucial issue in a rule-base inference formalism, especially when uncertainty is involved. Within the RIMER framework rule aggregation using an ER approach resolves the conflict and provides the aggregated conclusion. This will be explained in section 4.2.

The input for an antecedent attribute may not be available or may be only partially known. In the inference process, such incompleteness should be considered because it is related to the strength of a conclusion. This will be explained in section 5. Both complete and incomplete inference can be accommodated in a unified manner within the proposed RIMER framework.

\subsection{Time dependent rules}

Monitoring activities in a $\mathrm{SH}$ is a time dependent activity in the sense that being able to represent and reason about the order in which activities developed and their duration is essential for a correct diagnosis of the situations. Here we extend the RIMER framework with a temporal dimension. In addition, the system addresses specific areas of the house, rooms and their connecting areas, by the way of events, providing RIMER with spatio-temporal reasoning. For example there are states of being in a room (staying in a region)and events of passing from one room to another (a transition in between regions), $\left(\right.$ see $^{40}$ ).

\subsubsection{Time order}

Instantaneous events are associated with points in time. Here we assume a framework where events can be associated with a linear, discrete, totally-ordered, representation of time $\ldots t_{n-2}, t_{n-1}, t_{n}, t_{n+1}, t_{n+2}, \ldots$ over this time structure order relations like ' $<$ ' ('earlier than') and '=' ('simultaneous') can be defined, in a similar way as we do with other ordered structures like integers. Hence, the two possible relative positions between two time points $t_{1}$ and $t_{2}$ are that either one is earlier than the other or they are simultaneous. We will use classical logic ${ }^{41}$ connectives: $\wedge$ ("and"), $\vee$ ("or"), and $\neg$ ("not") to describe different possible temporal constraints over the temporal structure or the event occurrences associated with elements of that structure.

In our framework, a rule

$$
\text { IF }\left(E_{1} \wedge E_{2} \wedge C_{3} \wedge C_{4}\right) \text { THEN } A
$$

will be depicted slightly differently to represent that the conjunction in the antecedent of the rule is also including temporal order. For that purpose we introduce two symbols $\wedge$ and $A$ where $A \times B$ can be intuitively read as 'A is true and later B is true' and $A \quad A B$ can be intuitively read as ' $\mathrm{A}$ is true and simultaneously B is true'. They can be more formally defined as follows:

\author{
$A \times B$ if and only if \\ $\exists t_{1}, t_{2}$ such that: \\ $t_{1}<t_{2}$ and $\mathrm{A}$ is true at $t_{1}$ and $B$ is true at $t_{2}$
}


$A \quad A B$ if and only if

$\exists t$ such that:

$A$ is true at $t$ and $B$ is true at $t$

So, for example, lets say we use a predicate "oc$\operatorname{curs}(E)$ " to represent that event $E$ has been detected and "true $(C)$ " to represent that a condition $C$ is fulfilled in the current state of the system:

$$
\begin{aligned}
& \operatorname{IF}\left(\operatorname{occurs}\left(E_{1}\right) \text { Atrue }\left(C_{3}\right)\right) \times\left(\operatorname{occurs}\left(E_{2}\right) \text { Attrue }\left(C_{4}\right)\right) \\
& \text { THEN } A
\end{aligned}
$$

can be used to depict a scenario where it is meaningful that event $E_{1}$ occurs when condition $C_{3}$ is true and that is followed by the occurrence of event $E_{2}$ when conditions $C_{4}$ is true. Other notions of order between events occurrences can be defined based on the previous ones, for example:

$$
B \wedge A \text { if and only if } \neg(A \wedge B) \wedge \neg(A \wedge B)
$$

as we are assuming a totally ordered linear temporal structure.

This in turn has an effect on how rules are triggered. We depict a situation where events $E_{1}, E_{2}$, $E_{3}$, and $E_{4}$ have been recorded simultaneously with timestamp $t_{n}$ and events $E_{12}$, and $E_{13}$ have been recorded simultaneously with timestamp $t_{m}$.

Now let's assume we also have a rule with the following antecedent

$$
\operatorname{IF} \operatorname{occurs}\left(E_{3}\right) \quad \text { A } \operatorname{occurs}\left(E_{1}\right) \operatorname{THEN} \ldots
$$

This rule will be triggered by the events that occurred at time $t_{n}$, however a rule with the following antecedent:

$$
\operatorname{IF} \operatorname{occurs}\left(E_{3}\right) \text { A } \operatorname{occurs}\left(E_{12}\right) \operatorname{THEN} \ldots
$$

cannot be triggered. The following rule with a slightly different antecedent will be triggered by the events recorded in the database in the order given above:

$$
\operatorname{IF} \operatorname{occurs}\left(E_{3}\right) \times \operatorname{occurs}\left(E_{12}\right) \operatorname{THEN} \ldots
$$

Other operators can be defined in terms of those, for example ORnext can be defined as: $a$ ORnext $b={ }_{d e f}$
$a$ OR $b$ OR $(a$ ANDlater $b)$ OR $(b$ ANDlater $a)$

where OR is the classical logic disjunction.

Naturally in this framework the responsibility lies in the Knowledge Engineer who writes the rules using the correct operators to depict the meaningful situations that have to be captured. How events are registered in a particular time depends on the temporal granularity of the system. The usual computational trade offs apply here. The finer the granularity, the richer the depiction of the world but the heavier the computation. Whilst, the coarser the granularity, the easier becomes computing, but subtleties are lost. Here we assume that a sensor will keep its value when excited until the next reading when it is refreshed so there is no lost signal if the sensor is excited at a time between $t_{n}$ and $t_{n+1}$.

Actions can also have time attached. The time of the action is always the time when the rule advising a particular course of action is fired. Actions can be grouped in two main types:

1. recommendations to personnel: e.g.,"call/visit the occupant" if he/she has been inactive for a significant period of time during daytime, and

2. recording actions for the system itself: e.g., "assume occupant moved from reception to Living room" after detecting a sequence: motion in Reception, followed by occupant identified by tag detector in Reception to Living room door, followed by motion in the Living room.

\subsubsection{A richer temporal language}

Powerful temporal concepts can be built out of the language developed, which refer to time passing in a more succinct way.

A notion that " $n$ units of time have elapsed" (with $n \in\{1,2, \ldots\}$ and finite) can be defined as:

UnitsElapsed $(n)={ }_{\text {def }}$ event_occurrence $_{1} \times \ldots \times$ event_occurrence ${ }_{n}$

Timed event occurrences will be recorded each time 
sensors are read and equally, this process of reading sensors at regular intervals will be attached to a time stamp. Each sensor reading will produce at least one event detection as a special case of 'event_occurrence'. This can be 'no_event', i.e., the detection that no event has occurred.

Other definitions can be provided based on the previous concept:

UpperBoundUnitsElapsed $(n)={ }_{\text {def }}$

UnitsElapsed (1) $\vee \ldots \vee$ UnitsElapsed $(n)$

to express that at most $n$ units of time have elapsed.

$\operatorname{IsTrueAt}(P, n)=_{\text {def }}$ UnitsElapsed $(n) \AA P$

to express that condition $P$ is true when exactly $n$ units of time elapsed.

$\operatorname{IsTrueBefore}(P, n)={ }_{\text {def }}$

UpperBoundUnitsElapsed(n) AP

to express that condition $P$ is true at a time when less than or exactly $n$ units of time have elapsed. Notice we cannot introduce concepts like "sometimes in the future" or "always in the future" as they will imply translation into unbounded IF-THEN rules (we do not know exactly how far in the future the event occurs) but naturally we can use bounded temporal operators ${ }^{42}$ based on an interval $[a, b]$ (with $a<b$ and both $a$ and $b$ finite):

SometimeWithinF ut $\operatorname{Int}_{[a, b]}(P)={ }_{\text {def }}$ UnitsElapsed $(a) \times(U$ pperBoundUnitsElapsed $(b) \AA P)$

AlwaysWithinF utInt ${ }_{[a, b]}(P)={ }_{\text {def }}$

$\neg$ SometimeWithinF utInt $[a, b](\neg P)$

Similar (mirroring) definitions can be provided for the past fragment, for example to define: IsTrueAfter $(P, n)$, SometimeWithinPastInt $[a, b](P)$, and AlwaysWithinPastInt $[a, b](P)$.

\section{RIMER as a system to design Smart Homes}

There are two essential components in a rule-based SH system: a knowledge base and an inference engine. They are combined to infer useful conclusions from rules established by experts, e.g. from the caring personnel and facts obtained from sensors and other sources (e.g., databases).

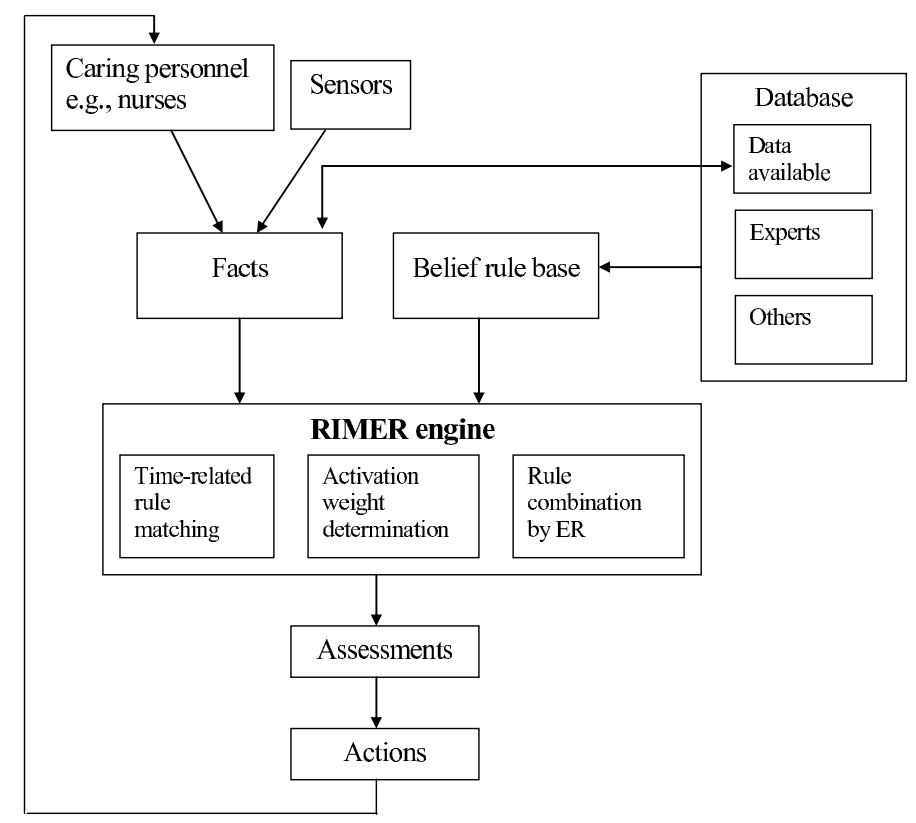

Fig. 2. General Architecture 
The general architecture of the system is illustrated in Figure 2. The knowledge base is defined and generated by the experts using a relational database. Some human or social aspects of the situation cannot be sensed or inferred by sensors or devices and in those cases expert judgment is needed to provide an approximation of the real situation with some degree of confidence. The rules-matching component then searches through a combination of facts to find those combinations that satisfy the antecedent of rules and select rules that should be fired. Time-related ordering is used to decide which of the rules, out of all that apply, have the highest priority and should be fired first and which of the rules cannot be used. The activation weight determination is used to calculate the matching degree of the facts to the IF part of the rules. These selected rules may conflict with each other if the IF parts of more than one rule are matched simultaneously. Then the rule combination scheme based on the ER algorithm is applied to get the final aggregated assessment which solves the rule conflicts. The database will be updated based on the new assessment and be fed into the rule-base and the new situation.

In the above sections, we have presented a general SH environment and explained how diagnosis in such cases is based on spatio-temporal considerations. Here we expand those considerations in relation to vagueness, imprecision and incompleteness of the information available. The design and implementation of rule-based SH systems for supporting decision making will be presented by using the RIMER framework which is based on DempsterShafer's theory of evidence ${ }^{43}$, decision theory 44,45 and fuzzy set theory $46,47,48,49$. Yang et al. ${ }^{22}$ proposed a new methodology for building a hybrid rule-base system using a belief structure and for inference in the rule-based system using the ER approach $50,51,25,26,27$.

\subsection{Belief Rule-Base}

A basic rule-base is composed of a collection of "IFTHEN" rules. To take into account a degree of belief in a consequent, attribute weights and a rule weight, a simple "IF-THEN" rule is extended to a so-called belief rule with all possible consequents associated with belief degrees. A belief rule, $R_{k}$, is defined as follows:

$$
\begin{array}{r}
R_{k}: \operatorname{IF}\left(X_{1} \text { is } A_{1}^{k}\right) \text { AND } \ldots \text { AND }\left(X_{T_{k}} \text { is } A_{k}^{T_{k}}\right) \\
\operatorname{THEN}\left\{\left(C_{1}, \beta_{1, k}\right), \ldots,\left(C_{N}, \beta_{N, k}\right)\right\}
\end{array}
$$

with rule weight $\theta_{k}$ and attribute weights $\delta_{1 k}, \delta_{2 k}, \ldots, \delta_{T_{k}, k}$ where $k \in\{1, \ldots, L\} . \quad \beta_{i, k}(i \in$ $\{1, \ldots, N\})$, such that $\sum_{i=1}^{N} \beta_{i, k} \leqslant 1$, is the belief degree to which $C_{i}$ is believed to be the consequent if in the $k^{\text {th }}$ rule the input satisfies the antecedent referential value vector $A^{k}=\left\{A_{1}^{k}, A_{2}^{k}, \ldots, A_{T_{k}}^{k}\right\} . L$ is the number of all belief rules used in the rule-base.

If $\sum_{i=1}^{N} \beta_{i, k}=1$, the $k^{\text {th }}$ belief rule is said to be complete; otherwise, it is incomplete. Note that $1-\sum_{i=1}^{N} \beta_{i, k}$ denotes the ignorance. And $\sum_{i=1}^{N} \beta_{i, k} \leqslant$ 0 denotes total ignorance about the output given in the $k^{\text {th }}$ rule. It is further assumed that $T$ is the total number of antecedent attributes used in the rule base.

Take for example the following belief rule, where values "High confidence", "Medium confidence", "Low confidence" and "None" are abbreviated as $(\mathrm{H}),(\mathrm{M}),(\mathrm{L})$ and $(\mathrm{N})$ respectively. The logical symbol $\lambda$ is replaced by ANDlater which the ASCII version used in the implementation.

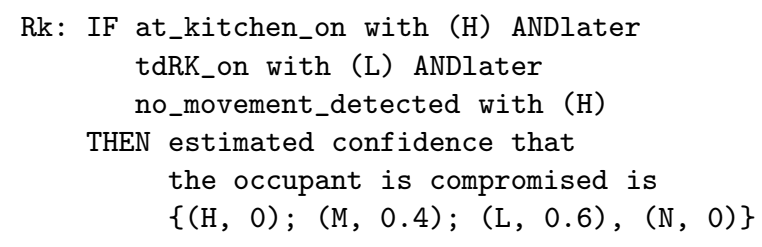

Here $\{(H, 0),(M, 0.4),(L, 0.6),(N, 0)\}$ is a belief distribution representation of the person's compromised health status (e.g. has fallen or fainted), indicating that we are $40 \%$ sure (the level of confidence) that the occupant has fainted is medium, and $60 \%$ sure that the occupant has fainted is low. In this belief rule, the total degree of belief is $0.4+0.6=1$, so the assessment is complete.

Remark 1: Note that in a rule-base, a referential value set can be a set of meaningful and distinctive evaluation levels for describing an attribute (here an event or a condition), e.g., it can be subjective linguistic terms. The referential value set for 'at_kitchen_on' is given by $A_{1}=\{H, M, L, N\}$. In a general rule-base, the attributes involved in each rule 
can be different in type, so their referential value sets may be also different in type.

Remark 2: Let $X=\left(X_{1}, X_{2}, \ldots, X_{T_{k}}\right)$, $A^{k}=\left(A_{1}^{k}, A_{2}^{k}, \ldots, A_{T_{k}}^{k}\right), \quad C=\left(C_{1}, C_{2}, \ldots, C_{N}\right)$, $\beta^{\mathbf{k}}=\left(\beta_{1, k}, \beta_{2, k}, \ldots, \beta_{N, k}\right)$, and $\delta=\left(\delta_{1}, \delta_{2}, \ldots, \delta_{T_{k}}\right)$.

$X$ is referred to as an input vector to the $k^{\text {th }}$ rule, $A^{k}$ a packet antecedent, $A_{i}^{k}\left(i=1,2, T_{k}\right)$ the $i^{t h}$ referential values of the packet antecedent $A^{k}, C$ the consequent vector, $\beta^{k}$ the vector of the belief degrees, and $\delta$ the attribute weights of all the $T$ antecedent attributes in the rule base. Suppose all $L$ rules are independent of each other, which means that the antecedent referential value vectors $\left\{A^{1}, \ldots, A^{L}\right\}$ are independent of each other.

In a traditional rule, the consequent is either $100 \%$ true or $100 \%$ false. Such a rule base has limited capacity in representing knowledge in a real world. A belief rule like the one given represents functional mappings between antecedents and consequents possibly with uncertainty. It provides a more informative and realistic scheme than a simple IF-THEN rule base when we need to consider uncertainty in knowledge representation.

\subsection{Inference using the evidential reasoning (ER) approach}

Given an input to the system, $U_{E C}=\left(U_{i} \mid i=\right.$ $1, \ldots, T)$, how can the rule base be used to infer and generate an output? $T$ is the total number of antecedent attributes in the rule base, $U_{i}(i=1, \ldots, T)$ is the $i^{\text {th }}$ antecedent attribute, which can be one of the following types: continuous, discrete, symbolic and ordered symbolic. Before the start of an inference process, the matching degree of an input to each referential value in the antecedents of a rule needs to be determined so that an activation weight for each rule can be generated. This is equivalent to transforming an input into a distribution of referential values by using belief degrees. The antecedent attributes involved in a rule for a $\mathrm{SH}$ system could be quantitative or qualitative, so that the input for each antecedent attribute may be different both in type and in scale. To facilitate data collection, it is desirable to acquire assessment information in a manner appropriate to a particular attribute.
Using the notations provided above, the activation weight of the $k^{\text {th }}$ rule, $w_{k}$, is calculated as ${ }^{22}$ :

$$
w_{k}=\frac{\theta_{k} \times \prod_{i=1}^{T_{k}}\left(\alpha_{i, k}\right)^{\bar{\delta}_{i}}}{\sum_{j=1}^{L} \theta_{j} \times \prod_{l=1}^{T_{k}}\left(\alpha_{l, j}\right)^{\bar{\delta}_{l}}}
$$

where:

$$
\bar{\delta}_{i}=\frac{\delta_{i}}{\max _{i=1, \ldots, T_{k}}\left\{\delta_{i}\right\}} \text { so } 0 \leqslant \bar{\delta}_{i} \leqslant 1
$$

Here $\alpha_{i, k}\left(i=1, \ldots, T_{k}\right)$, called the individual matching degree, is the degree of belief to which the input for the $i^{\text {th }}$ antecedent attribute belongs to its referential value $A_{i}^{k}$ in the $k^{\text {th }}$ rule, $\alpha_{i, k} \geqslant 0$ and $\sum_{i=1}^{T_{k}} \alpha_{i, k} \leqslant 1$. The set $\alpha_{k}=\prod_{i=1}^{T_{k}}\left(\alpha_{i, k}\right)^{\bar{\delta}_{i}}$, is called the combined matching degree. Note that $0 \leqslant w_{k} \leqslant 1(k=1, \ldots, L)$ and $\sum_{i=1}^{L} w_{i}=1$. Also note that $w_{k}=0$ if the $k^{\text {th }}$ rule is not activated.

Having determined the activation weight of each rule in the rule base, the ER approach ${ }^{25,26}$ ) can be directly applied to combine the rules and generate final conclusions. Suppose the outcome of the combination yields the following

$$
O(U)=\left\{\left(C_{j}, \beta_{j}\right) \mid j=1, \ldots, N\right\}
$$

The outcome expressed by equation ( 3 ) reads that if the input is given by $U_{E C}=\left(U_{i} \mid i=1, \ldots, T\right)$ then the consequent is " $C_{1}$ to a degree of $\beta_{1}$ ", " $C_{2}$ to a degree of $\beta_{2}$ ",$\ldots$, and " $C_{N}$ to a degree of $\beta_{N}$ ". Using the analytical format of the ER algorithm ${ }^{22}$ the combined belief degree in $C_{j}$ can be generated as follows:

$$
\beta_{j}=\frac{\mu \times\left[M_{1}-M_{2}\right]}{1-\mu \times\left[\prod_{k=1}^{L}\left(1-w_{k}\right)\right]}
$$

with

$$
\begin{gathered}
M_{1}=\prod_{k=1}^{L}\left(w_{k} \beta_{j, k}+1-w_{k} \sum_{j=1}^{N} \beta_{j, k}\right) \\
M_{2}=\prod_{k=1}^{L}\left(1-w_{k} \sum_{j=1}^{N} \beta_{j, k}\right) \\
\mu=\left[\sum_{j=1}^{N} M_{1}-(N-1) M 2\right]^{-1}
\end{gathered}
$$

where $j=1, \ldots, N$ and $w_{k}$ is calculated by equation (2).

This permits the computation of the belief degree distribution. 


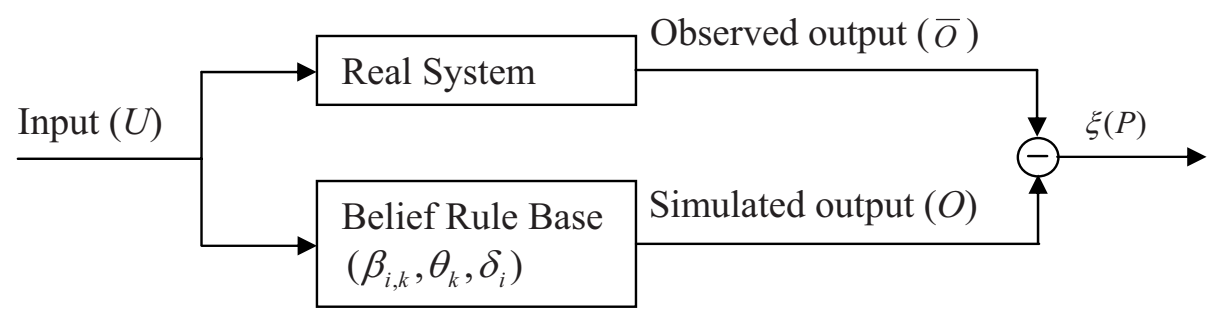

Fig. 3. Illustration of Optimal LearningProcess

\subsection{Optimal method for training belief rule bases in RIMER}

Although it is possible to establish a belief rule base by extracting knowledge from experts, the performance of the system can be improved if the rules are fine tuned through learning from available historical data.

The adjustable parameters of a rule base are belief degrees $\left(\beta_{1, k}, \beta_{2, k}, \ldots, \beta_{N, k}\right)$, rule weights $\theta_{k}$ for $k=1, \ldots, L$ and attribute weights $\left(\delta_{1}, \delta_{2}, \ldots, \delta_{T_{k}}\right)^{22}$.

Figure 3 sketches the process of training a belief rule base, where $U$ is a given input, $\bar{O}$ the corresponding observed output, either measured using instruments or assessed by experts, $O$ the simulated output generated by the belief rule based system, $\xi(P)$ the difference between $\bar{O}$ and $O$, and

$P=\left(\beta_{i, k}, \theta_{k}, \delta_{j} ; i=1, \ldots, N ; k=1, \ldots, L ; j=1, \ldots, T\right)$

are the adjustable parameters. The objective of the training is to minimize the difference $\xi(P)$ by adjusting the parameters $P$. This objective is difficult to achieve manually even by experts, however there are computer algorithms available to solve the problem. Yang et al. ${ }^{53}$ and Liu et al. ${ }^{54}$ discuss in more detail how the problems can be constructed for different types of output and algorithms applied to solve them. By using the optimal learning methods for training the belief rules, the belief rule based system can learn from SH data the relationship between event/condition and the possible action. It has also been demonstrated ${ }^{53}$ that learning could start with a random rule base and therefore prior-knowledge does not have to be provided.

\section{Case study}

\subsection{Problem description}

Consider a scenario where there is a potential hazard related to the SH occupant and different procedures can be put in place to prevent the hazard or to react if there is an indication that the occupant may be at risk. Absence of motion for a period which is considered unusually prolonged combined with the occupant's location and the time of the day can be considered an indication that the occupant may be at risk. It is in the interest of the occupant that the system reacts preemptively, e.g., initiate direct contact with the occupant for confirmation. If contact is made, consider situation to be normal, otherwise trigger a pre-established emergency procedure.

However, there could be ambiguous scenarios, e.g., when the occupant is standing under doors for a prolonged period of time, which results in absence of motion in the rooms. If the occupant remains motionless under a door connecting two rooms: is it likely that the person has fallen, fainted, is resting or is talking to someone else in another room?

First we give a general schema without considering uncertainty representation and then we show how this information can be dealt with by following the RIMER approach.

We will assume that the occupant has 'fainted', and no motion sensors have been activated for a considerable length of time. Below we exemplify with the door connecting the reception area with the Living room. Similar groups of schema rules should be in the Knowledge Base with respect 
to the other doors. Using the operator "ANDlater" introduced in Section 3, which checks the order of arrival of the knowledge atoms to the Knowledge base, an IF-THEN rule can be given as follows:

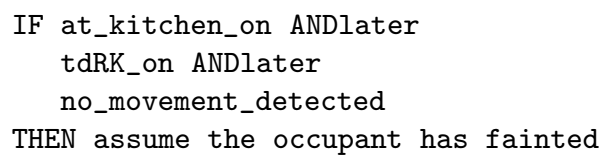

The above mentioned IF-THEN rule is the rule without considering the uncertainties involved. The following sub-sections will extend this rule in such a way that the belief rule can cover different levels of uncertainty.

\subsection{Referential Grades of the Antecedents and Actions}

The number of referential grades used for each antecedent decides the size of the rule base. If the number is too large, there will be too many rules, and the inference process will be more demanding. If it is too small, the grades may not be able to cover the value range of an antecedent attribute. This is especially true for a conventional rule base. Normally 3 to 9 referential grades are used. The number of referential grades for a consequent attribute is also comparable to those of the antecedent attributes. Because of the presence of uncertainty in the system, the observation or confidence for each input state may be uncertain. This example uses the input states ("at_kitchen_on", "tdRK_on" and no motion sensors activated for a considerable length of time) to predict if a occupant has fainted. The necessary combinations of states "at_kitchen_on" and "tdRK_on" are based on the monitoring from the equipment and the opinion given by experts. This judgment is inevitably associated with uncertainties due to inability to provide precision all the time about the sensors, or the lack of information, or the vagueness and ambiguity in the meaning of some attributes and their assessment. So the input states could be directly associated to a distribution using their referential linguistic grades with the degrees of belief based on subjective judgments. For illustration purposes, each of these input states and the output state (confidence to which the person may have a health problem, e.g., had fainted) are defined as having values of High (H), Medium (M), Low (L) or None $(\mathrm{N})$. That is, the grades for "at_kitchen_on" are:

$$
A_{1}^{k} \in\{H, M, L, N\}, k \in\{1, \ldots, L\}
$$

similarly we also use those grades for "tdRK_on" $\left(A_{2}^{k}\right)$ and for "no_movement" $\left(A_{3}^{k}\right)$. Each state is assessed into a belief distribution representation of these four values. For example if the assessment of "at_kitchen_on" is

$$
\left\{\left(H, \beta_{1}\right),\left(M, \beta_{2}\right),\left(L, \beta_{3}\right),\left(N, \beta_{4}\right)\right\}
$$

implies the possibility of "at_kitchen_on" being triggered and also the confidence level of "at_kitchen_on" if it occurs, where $\beta_{i}(i=1, \ldots 4$, represents the degree of confidence in a particular belief.

If a "NOT" connective is used in the rule it has the semantics of "failing to detect". This can be used in two ways, a) the corresponding sensor was not activated (for example, no movement was produced in the kitchen so that sensor will stay off) and b) even when there was an activation, the context advised not to consider it as such (for example, a sensor signal is dismissed because other aspects of the scenario lead to the belief that it is malfunctioning). This can be also rephrased by stating that the event is assessed as "None" with belief degree 1. For example, for "None", if the occupant is believed to be at home with confidence graded "None", then it means the occupant is absent from home.

For the consequent attribute, four cases are considered, i.e., High $(\mathrm{H})$ possibility that the occupant has fainted; Medium (M) possibility that the occupant has fainted; Low (L) possibility that the occupant has fainted and, in the most positive scenario, None $(\mathrm{N})$ meaning that nothing significant has happened. So this example uses the input states to predict "if a occupant has fainted" in terms of qualitative linguistic terms.

\subsection{Defining the Rule Base}

Space constraints do not allow us to give a full account of all the rules of all knowledge bases, instead we focus on how to attach the representation of uncertainty to a rule related to the detection of our reference scenario. Using the linguistic grades, one of 
the conventional rules for predicting "if a occupant has fainted" is given:

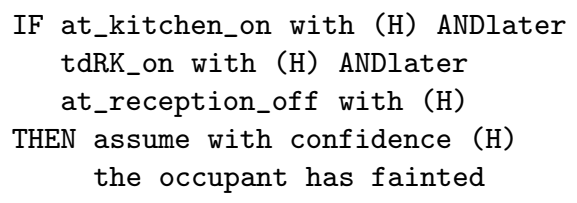

The rule has only one consequent with a belief degree being always exactly one. Such conventional IF-THEN rules cannot capture the continuous relationships between the antecedent and the consequents. Therefore, the expert system may not be able to accurately reflect the real context, in this case what happens in the SH. We can extend the rules using the belief structure to provide better flexibility and versatility which are needed to model human reasoning more adequately. The definitions of the extended rules using linguistic terms with the consequents having the dedicated degrees of belief are given in Table 1. Note that in the table we utilize at_reception_off with high confidence to imply "no_movement_detected" in the observed time period. There are sixteen rules according to the number of linguistic terms in the input states. The degrees of belief in the consequents were assigned by the researchers as a result of the observation of the given expert judgments. In a more systematic scheme, the belief degrees could be trained using expert judgments as test data and may also be updated once new evidence becomes available. The rule base can be applied to both discrete and continuous reasoning processes.

The rule given in this section is represented by the 2 nd row of the table.

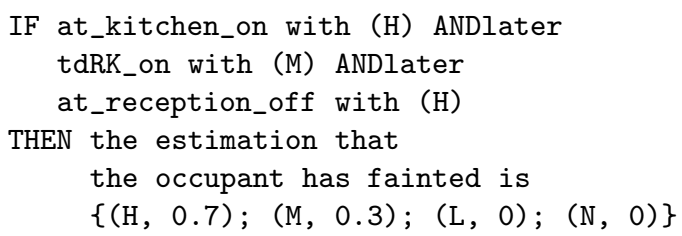

Here (H, 0.7); (M, 0.3); (L, 0); (N, 0) means the system has a degree of confidence of $70 \%$ that "the occupant has fainted" occurred with high possibility, and of $30 \%$ that "the occupant has fainted" occurred with medium possibility. The example aims to determine a confidence degree to which the expert believes that the occupant may have a health problem so that the emergency procedure can be applied. For example, if the final output for "if a occupant has fainted" is with low or zero confidence, then no further actions are needed. If the final output for "if a occupant has fainted" is with medium or high confidence, the possible further actions can be applied.

Table 1. Rule-base table

\begin{tabular}{|l|c|c|c|c|}
\hline Rule & \multicolumn{3}{|c|}{ Antecedents } & Consequent \\
\hline & $\begin{array}{c}\text { at_kitchen } \\
\text { on }\end{array}$ & tdRK & $\begin{array}{c}\text { at_reception } \\
\text { off }\end{array}$ & Belief distribution \\
\hline 1 & H & H & H & $\{(\mathrm{H}, 0.9),(\mathrm{M}, 0.1),(\mathrm{L}, 0),(\mathrm{N}, 0)\}$ \\
\hline 2 & H & M & H & $\{(\mathrm{H}, 0.7),(\mathrm{M}, 0.3),(\mathrm{L}, 0),(\mathrm{N}, 0)\}$ \\
\hline 3 & H & L & H & $\{(\mathrm{H}, 0),(\mathrm{M}, 0.4),(\mathrm{L}, 0.6),(\mathrm{N}, 0)\}$ \\
\hline 4 & H & N & H & $\{(\mathrm{H}, 0),(\mathrm{M}, 0),(\mathrm{L}, 1),(\mathrm{N}, 0)\}$ \\
\hline 5 & M & H & H & $\{(\mathrm{H}, 0.7),(\mathrm{M}, 0.3),(\mathrm{L}, 0),(\mathrm{N}, 0)\}$ \\
\hline 6 & M & M & H & $\{(\mathrm{H}, 0.3),(\mathrm{M}, 0.7),(\mathrm{L}, 0),(\mathrm{N}, 0)\}$ \\
\hline 7 & M & L & H & $\{(\mathrm{H}, 0),(\mathrm{M}, 0.3),(\mathrm{L}, 0.7),(\mathrm{N}, 0)\}$ \\
\hline 8 & M & N & H & $\{(\mathrm{H}, 0),(\mathrm{M}, 0),(\mathrm{L}, 1),(\mathrm{N}, 0)\}$ \\
\hline 9 & L & H & H & $\{(\mathrm{H}, 0),(\mathrm{M}, 0.4),(\mathrm{L}, 0.6),(\mathrm{N}, 0)\}$ \\
\hline 10 & L & M & H & $\{(\mathrm{H}, 0),(\mathrm{M}, 0.3),(\mathrm{L}, 0.7),(\mathrm{N}, 0)\}$ \\
\hline 11 & L & L & H & $\{(\mathrm{H}, 0),(\mathrm{M}, 0),(\mathrm{L}, 0),(\mathrm{N}, 1)\}$ \\
\hline 12 & L & N & H & $\{(\mathrm{H}, 0),(\mathrm{M}, 0),(\mathrm{L}, 0),(\mathrm{N}, 1)\}$ \\
\hline 13 & N & H & H & $\{(\mathrm{H}, 0),(\mathrm{M}, 0),(\mathrm{L}, 0),(\mathrm{N}, 1)\}$ \\
\hline 14 & $\mathrm{~N}$ & M & H & $\{(\mathrm{H}, 0),(\mathrm{M}, 0),(\mathrm{L}, 0),(\mathrm{N}, 1)\}$ \\
\hline 15 & N & L & H & $\{(\mathrm{H}, 0),(\mathrm{M}, 0),(\mathrm{L}, 0),(\mathrm{N}, 1)\}$ \\
\hline 16 & N & N & H & $\{(\mathrm{H}, 0),(\mathrm{M}, 0),(\mathrm{L}, 0),(\mathrm{N}, 1)\}$ \\
\hline
\end{tabular}




\subsection{Belief rule inference using the evidential reasoning (ER) approach}

Using the rule-base in Table 1 and the RIMER inference scheme, the consequent estimate is generated. Following we explore some possible combinations of values to see how the system reacts.

Case 1: The input for "at_kitchen_on" is given by the expert with a belief distribution, for example: $\{(\mathrm{H}, 0.9) ;(\mathrm{M}, 0.1) ;(\mathrm{L}, 0) ;(\mathrm{N}, 0)\}$ which means that the experts are $90 \%$ sure that "at_kitchen_on" occurred with high confidence, $10 \%$ that "at_kitchen_on" occurred with medium confidence. The input for "tdRK_on" is given by the expert with a belief distribution, for example: $\{(\mathrm{H}, 0.9) ;(\mathrm{M}, 0.1)$; $(\mathrm{L}, 0) ;(\mathrm{N}, 0)\}$ Moreover, there is no motion sensors activated for a considerable length of time, represented as: $\{(\mathrm{H}, 1) ;(\mathrm{M}, 0) ;(\mathrm{L}, 0)$; $(\mathrm{N}, 0)\}$ i.e., $100 \%$ sure that nothing happened. In summary, it is represented as:

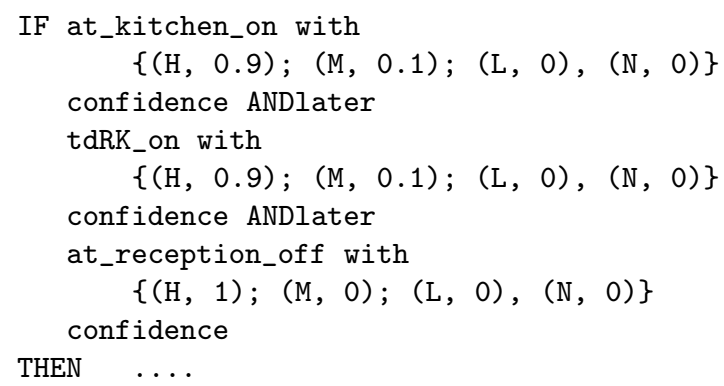

The output is implemented as in the following steps:

Step 1: Transform the input. Here the input is given as a distribution using linguistic terms with the degrees of belief based on subjective judgments. Each belief is the individual matching degree of the input to the linguistic value. For example, the matching degree of the input to the linguistic value "High" of "at_kitchen_on" is 0.9 , and 0.8 for "Medium", etc.

Step 2: Calculate rule activation weight. The activation weights $w_{k}$ for all the sixteen rules $R_{k}(k=1, \ldots, 16)$ are generated by using equation ( 2 ) in Section 4.1 by $w_{1}=0.81, w_{2}=0.09, w_{3}=0, w_{4}=$ $0, w_{5}=0.09, w_{6}=0.01, w_{7}=0, w_{8}=$ $0, w_{9}=0, w_{10}=0, w_{11}=0, w_{12}=$ $0, w_{13}=0, w_{14}=0, w_{15}=0, w_{16}=$ 0 , respectively. For example, $\alpha_{1}=$ $\prod_{i=1}^{T_{1}}\left(\alpha_{1, i}\right)^{\bar{\delta}_{i}}=0.9 \times 0.9 \times 1=0.81$, see explanation for equation (2). Here $T_{1}$ is the number of antecedent attribute in the $i^{\text {st }}$ rule and $T_{1}=3$. Following the same steps, $\alpha_{i}$ can be obtained, and then using equation (2), $\omega_{i}(i=1, \ldots, 16)$ can be obtained. Note that the attribute weights and the rule weights are assumed to be one.

Step 3: Combine activated rules. The ER approach ${ }^{26}$ is employed to combine the activated rules. Using the IDS software, the activated rules can be combined to yield the following outcome (see Case 1 in Figure 4):

$$
\begin{aligned}
& O(U(1))=\left\{\left(C_{j}, \beta_{j}\right), j=1, \ldots, 4\right\}= \\
& \{(\mathrm{H}, 0.8969) ;(\mathrm{M}, 0.1031) ;(\mathrm{L}, 0) ;(\mathrm{N}, 0)\}
\end{aligned}
$$

where $\beta_{j}$ is given by equation (4), and $\left(C_{1}, C_{2}, C_{3}, C_{4}\right)=(\mathrm{H}, \mathrm{M}, \mathrm{L}, \mathrm{N})$.

which means that we are $89.69 \%$ sure that the occupant has fainted with high confidence, $10.31 \%$ sure that the occupant has fainted with medium confidence, $0 \%$ sure that nothing happens for the occupant. 

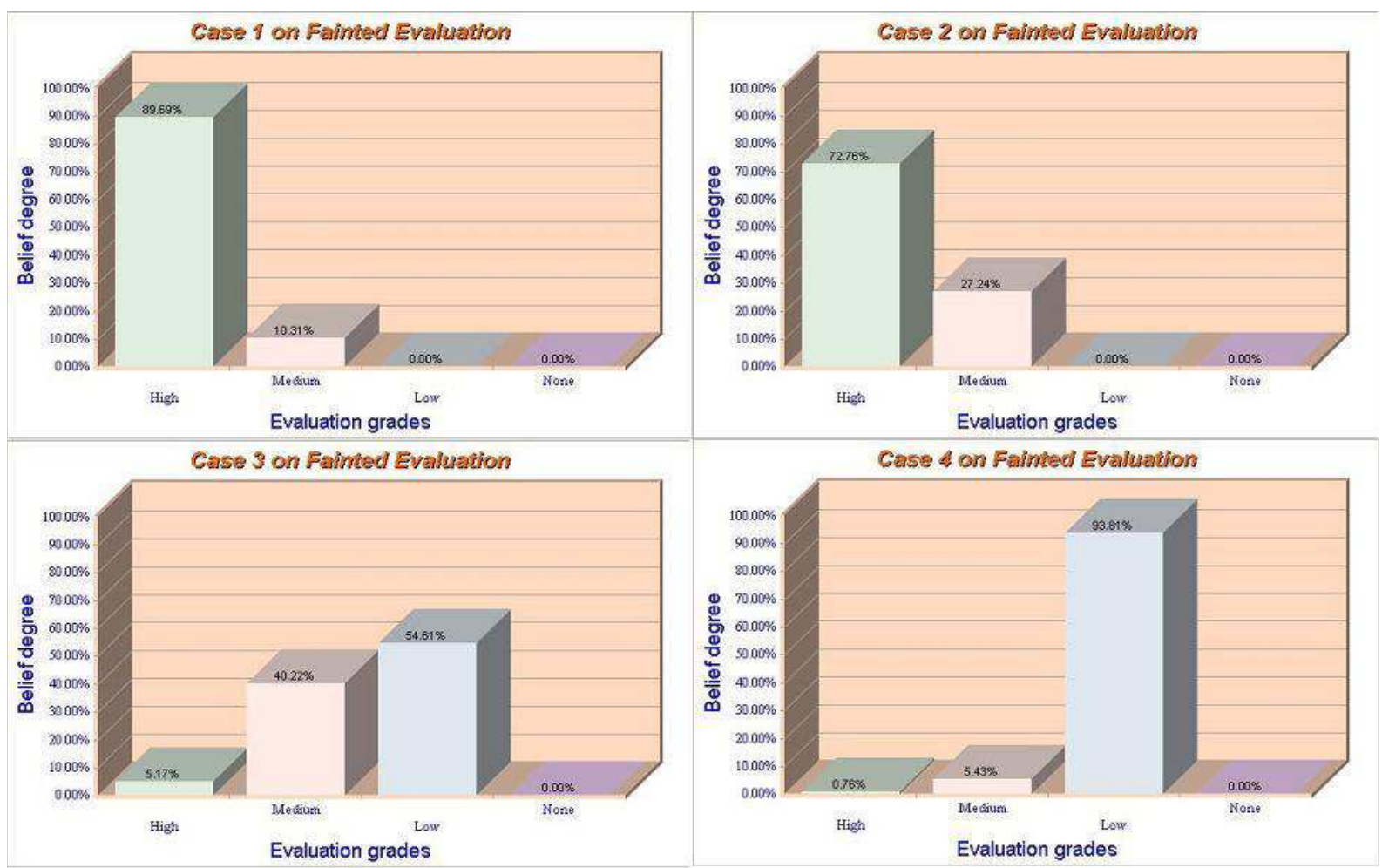

Fig. 4. Belief distribution for Case1: at_kitchen_on (H), tdRK $(\mathrm{H})$, at_reception_off $(\mathrm{H})$; Case2: at_kitchen_on $(\mathrm{H})$, tdRk (M), at_reception_off $(\mathrm{H})$; Case3: at_kitchen $(\mathrm{H})$, tdRK (L), at_reception_off $(\mathrm{H})$; Case4: at_kitchen_on(L), tdRk (L), at_reception_off $(\mathrm{H})$

Case 2: Suppose that the input information is give as follows:

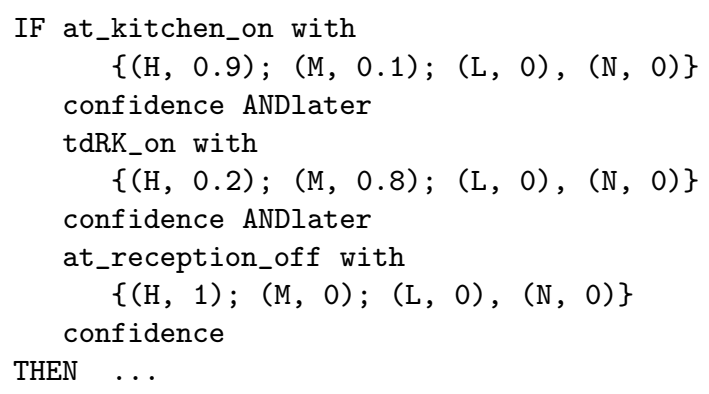

Following similar steps than in Case 1, the system output is (see Case 2 in Figure 4): $O(U(2))=\left\{\left(C_{j}, \beta_{j}\right), j=1, \ldots, 4\right\}=$ $\{(\mathrm{H}, 0.7276) ;(\mathrm{M}, 0.2724) ;(\mathrm{L}, 0) ;(\mathrm{N}, 0)\}$
Case 3: Suppose that the input information is give as follows:

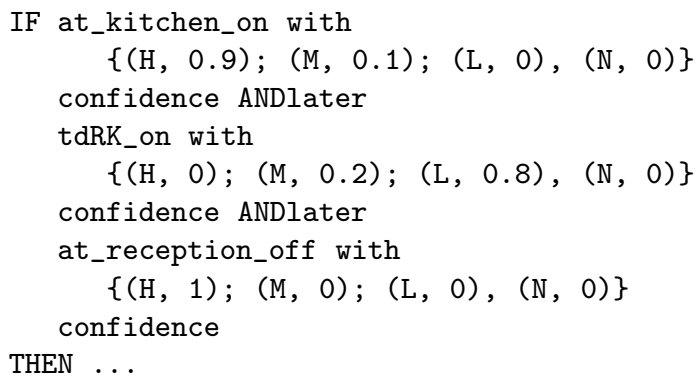

Following similar steps than in Case 1, the system output is (see Case 3 in Figure 4): $O(U(3))=\left\{\left(C_{j}, \beta_{j}\right), j=1, \ldots, 4\right\}=$ $\{(\mathrm{H}, 0.0517) ;(\mathrm{M}, 0.4022) ;(\mathrm{L}, 0.5461) ;(\mathrm{N}, 0)\}$ 
Case 4: Suppose that the input information is given as follows:

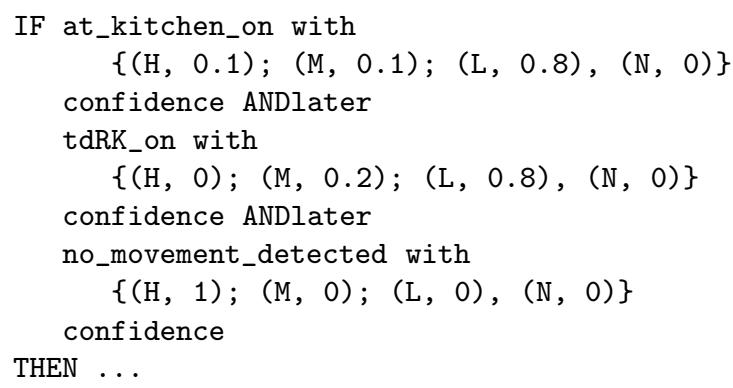

Following similar steps than in Case 1, the system output is (see Case 4 in Figure 4): $O(U(4))=\left\{\left(C_{j}, \beta_{j}\right), j=1, \ldots, 4\right\}=$ $\{(\mathrm{H}, 0.0076) ;(\mathrm{M}, 0.0543) ;(\mathrm{L}, 0.9381) ;(\mathrm{N}, 0)\}$

We may notice that firstly, if the activation weight of a rule is equal to 0 (e.g., $\left.w_{3}=0\right)$, then the weight and the belief degree of this rule will have no influence on the final output; If the activation weight of a rule is not equal to 0 , then the weight and the belief degrees of this rule will affect the final output. The degree to which the final output can be affected is determined by the magnitude of the activation weight and the belief degrees. The logic behind the approach is that if the consequent in the $k^{\text {th }}$ rule includes $C_{i}$ and the $k^{\text {th }}$ rule is activated, then the overall output must be $C_{i}$ to a certain degree. As shown from the examples, the degree is measured by both the degree to which the $k^{\text {th }}$ rule is important to the overall output and the degree to which the antecedents of the $k^{t h}$ rule are activated by the actual input. The distribution assessment provides a panoramic view about the output status, from which one can see the variation between the original output and the revised output on each linguistic term. From the above examples we may notice that if vague information coexists with ignorance or incompleteness caused due to the evidence not being strong enough to make simple true or false judgments but with degrees of belief, the RIMER system can provide a flexible and effective way to represent and deal with such uncertain assessment information to arrive at rational conclusions.

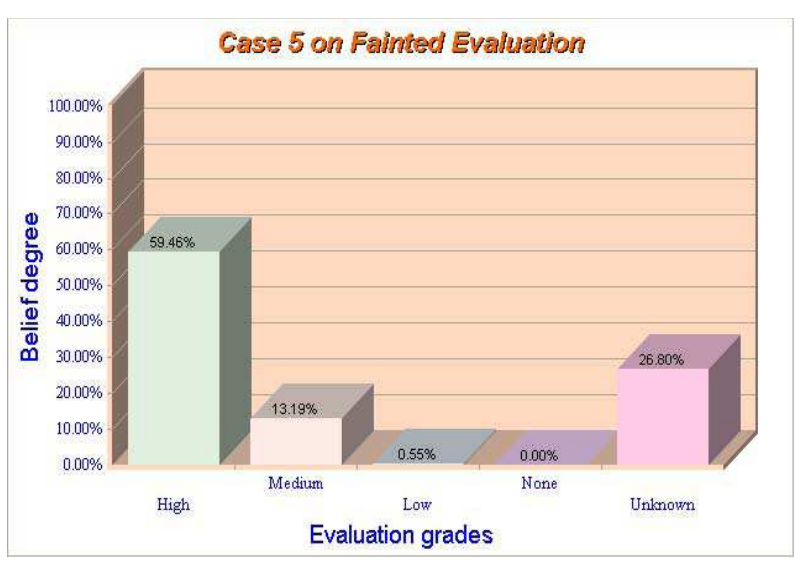

Fig. 5. Belief distribution for at_kitchen_on $(\mathrm{H}), \operatorname{tdRk}(\mathrm{H})$, at_reception_off (incomplete)

\subsection{Inference based on incomplete input information}

Assume one of the main events in the antecedent of our IF-THEN rule is not known. For example, we know "at_kitchen on" with high confidence and "tdRK_on" with relatively high confidence, but we only have partial evidence that after some time units the person is not moving, i.e., we are not $100 \%$ sure, lets say the belief distribution is $(\mathrm{H}, 0.7)$; $(\mathrm{M}, 0)$; $(\mathrm{L}$, $0),(\mathrm{N}, 0)$. This could be due a sensor fault, expert's inability to provide precise judgments, or information not being transmitted properly over the network from the SH to the computing centre. We can still infer the result based on the rule-base. To illustrate how incomplete input can be dealt with in the inference methodology, in the above case study we use the following input information:

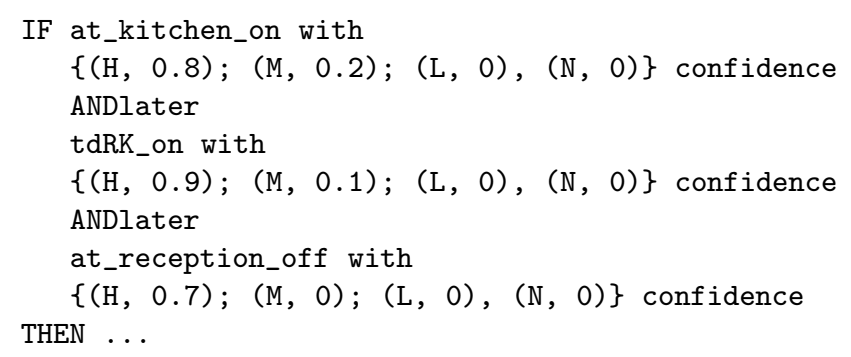

Notice that the experts are only $70 \%$ certain that there is no movement detected (at_reception_off). In other words, the degree of ignorance is 0.3 . Due to 
the assumed incomplete input for, we need to update the belief degree of the relevant rules to reflect the incompleteness. If we apply our methodology as in the previous section then the conclusion from the system will be: (high, 0.5946); (medium, 0.1319); (low, 0.0055); (nothing, 0); (unknown, 0.2680) where "Unknown" in the above result means that the output is also incomplete due to the incomplete input (see Figure 5). Hence, both complete and incomplete inference can be accommodated in a unified manner within the proposed RIMER framework.

\section{Conclusions}

Smart Homes are considered as one way to decentralize the delivery of health care to the population. Although there are many possible applications, current SH settings are devoted to their use as a protective environment for vulnerable people where their daily activities can be monitored in order to prevent hazards, profile behavior to facilitate diagnostic tasks or to react to a problem.

Although the hardware and communication layers of these systems are available and have been considered at length in the literature, the software side of these systems have not made similar advances. This article shows how the combination of spatiotemporal and uncertainty reasoning (using the ER approach) can improve the ways in which SHs can be designed. We emphasized the importance of embedding into the system spatio-temporal knowledge in order to assess how the diagnosis of a situation is dependent on where it is occurring and on the order and duration of the events that lead to that situation. We have introduced the temporal operators "ANDlater" and "ANDsim", upon which a richer temporal language can be constructed. In the current implementation the operators are parsed before run time and IF-THEN rules produced with antecedents using $>,=$ and Boolean operators. Future work will be oriented towards including the more complex temporal operators in the final language interpreted by the inference engine. This enriches the way a decision support system can help to diagnose if the situation deserves intervention. Equally important is the ability to cope with uncertainty due to the lack of complete information or the unreliability of some of the technical components involved (e.g., networks and sensors). The resulting system can represent knowledge about the activities in a SH. The knowledge can then be used to detect a problem, which is occurring or is likely to occur, to infer about the nature of the problem and to advise with intervention procedures through which the problem can be resolved in order to ameliorate the situation. This intervention can be achieved by controllable devices in the house or manually by carers (e.g., nurses, security personnel, relatives).

So far SHs systems design has neglected these issues and the focus of the literature has been much more on the possible benefits of the associated technology than on how to achieve them. Here we provide a solid foundation for the development of these kind of systems. Much remains to be done, particularly regarding verification of the reasoning process in a practical setting where the occupant is undertaking normal ADL. However the case studies developed can bring to the attention of the future developers the importance of these concepts and the need to provide systems with solid theoretical foundations.

In a belief rule based system, while human expert knowledge is used to construct a roughly correct belief rule base (a potential weakness), learning can help to fine tune system performance if the system input-output data are available. We believe that reasoning with fine-tuned logical rules is more acceptable to human users than the recommendations given by a black box system (e.g. neural network), because such reasoning is comprehensible, provides explanations, and can be verified automatically and validated by human inspection. It also increases confidence in the system, and may help to discover important relationships and combinations of features ${ }^{53}$.

In conclusion, combining spatio-temporal reasoning with uncertainty reasoning captures essential concepts that we believe should be considered when designing a SH system. Equally these characteristics are important to other applications of Ambient Intelligence, whether the environment under consideration is a hospital, a manufacturing unit or a street. 


\section{Acknowledgments}

The authors are very grateful to Djilali Boudiaf from Atlantic Press for the assistance provided on formatting of the paper.

\section{References}

1. M. Andersson, K. Clough, I. J., L. Kennedy, and D. Preston. The impact of e-health and assistive technologies on healthcare. Technical report, September 2005. www.tehip.org.uk.

2. Department of Health. Improving chronic disease management. Technical report, March 2004. www.dh.gov.uk.

3. J. C. Augusto and D. J. Cook. Ambient Intelligence: applications in society and opportunities for AI. 20th International Joint Conference on Artificial Intelligence (IJCAI-07). 2007.

4. J. C. Augusto and P. McCullagh. Ambient intelligence: Concepts and applications. In Computer Science and Information Systems, volume 4, pages 1-28. ComSIS Consortium, 2007.

5. J. C. Augusto and Daniel Shapiro, editors. Advances in Ambient Intelligence, volume 164 of Frontiers in Artificial Intelligence and Applications (FAIA) series. IOS Press, 2007.

6. C. Ramos, J. C. Augusto, and D. Shapiro. Ambient intelligence - the next step for artificial intelligence (guest editors' introduction to the special issue on ambient intelligence). IEEE Intelligent Systems, 23(2):15-18, Mar/Apr 2008.

7. J. C. Augusto. Ambient Intelligence: the Confluence of Ubiquitous/Pervasive Computing and Artificial Intelligence, pages 213-234. Intelligent Computing Everywhere. Springer London, 2007.

8. M. Weiser. The computer for the 21 st century. Scientific American, 265(3):94-104, 1991.

9. D. J. Cook and S. K. Das. Smart Environments: Technology, Protocols and Applications. WileyInterscience, 2005.

10. J. C. Augusto, H. Wang, and J. Liu. Situation assessment during disaster management. International Journal of Computational Intelligence System, 1(3):237247, 2008.

11. J. C. Augusto and C. D. Nugent, editors. Designing Smart Homes: The role of Artificial Intelligence, volume 4008 of Lecture Notes in Artificial Intelligence. Springer Verlag, 2006.

12. P. Tang and T. Venables. Smart homes and telecare for independent living. J Telemed Telecare, 6(1):814, 2000.

13. V. Rialle, F. Duchene, N. Noury, L. Bajolle, and J. Demongeot. Health smart home: information technology for patients at home. Telemed J E Health, 8(4):395409, 2002.

14. J. C. Augusto, C. D. Nugent, S. Martin, and C. Olphert. Towards personalization of services and an integrated service model for smart homes applied to elderly care. In S. Giroux and H. Pigot, editors, Proceedings of International Conference on Smart Homes and health Telematic, pages 151-158. IOS Press, 2005. July 4-6.

15. Edited by: S. Giroux and H. Pigot, editors. From Smart Homes to Smart Care, Proceedings of ICOST 2005, Volume 15 of Assistive Technology Research Series. IOS Press, June 2005.

16. G. Williams, K. Doughty, and D. Bradley. A systems approach to achieving carernet - an integrated and intelligent telecare system. IEEE Trans Inf Technol Biomed, 2(1):1-9, 1998.

17. A. Sixsmith. An evaluation of an intelligent home monitoring system. Journal of Telemedicine and Telecare, 6(2):63-72, 2000.

18. E. H. Shortliffe. Computer-based medical consultations: Mycin. Technical report, $1976 . \quad$ http://smi.stanford.edu/smiweb/research/details.jsp?PubId=12.

19. R. Dechter, I. Meiri, and J. Pearl. Temporal constraint networks. Artificial Intelligence, 49:61-95, 1991.

20. R. Bellazzi, P. Magni, and G. DeNicolao. Bayesian analysis of blood glucose time series from diabetes home monitoring. IEEE Trans Biomed Eng, 47(7):971-5, 2000.

21. D. Patterson, D. Fox, H. Kautz, and M. Philipose. Fine-grained activity recognition by aggregating abstract object usage. In Kiyoshi KIYOKAWA, editor, Proc. of IEEE 9th International Symposium on Wearable Computers, pages 220-221. IEEE, 2005. Oct 18-21.

22. J. B. Yang, J. Liu, J. Wang, H. S. Sii, and H. W. Wang. A belief rule-base inference methodology using the evidential reasoning approach - RIMER. IEEE Transactions on Systems, Man, and Cybernetics, Part A: Systems and Humans, 36(2):266-285, 2006.

23. J. C. Augusto and C. D. Nugent. The use of temporal reasoning and management of complex events in smart homes. In R. López de Mántaras and L. Saitta, editors, Proceedings of European Conference on Artificial Intelligence (ECAI 2004), pages 778-782. IOS Press (Amsterdam, The Netherlands), 2004. August, 22-27.

24. J. C. Augusto and C. D. Nugent. A new architecture for smart homes based on adb and temporal reasoning. In D. Zhang and M. Mokhtari, editors, Toward a Human Friendly Assistive Environment (Proceedings of 2 nd International Conference On Smart homes and health Telematic), pages 106-113. IOS Press, 2004. Assistive Technology Research Series, Volume 14. 
September 15-17.

25. J. B. Yang. Rule and utility based evidential reasoning approach for multi-attribute decision analysis under uncertainties. European Journal of Operational Research, 131(1):31-61, 2001.

26. J. B. Yang and D. L. Xu. On the evidential reasoning algorithm for multiple attribute decision analysis under uncertainty. IEEE Transactions on Systems, Man, and Cybernetics (Part A: Systems and Humans), 32(3):289-304, 2002.

27. J. B. Yang and D. L. Xu. Nonlinear information aggregation via evidential reasoning in multi-attribute decision analysis under uncertainty. IEEE Transactions on Systems, Man, and Cybernetics (Part A: Systems and Humans), 32(3):376-393, 2002.

28. R. Sun. Robust reasoning: integrating rule-based and similarity-based reasoning. Artificial Intelligence, 75(2):241-295, 1995.

29. S. Parson. Current approaches to handling imperfect information in data and knowledge bases. IEEE Transactions on Knowledge and Data Engineering, 8(3):353-372, 1996.

30. J. Hodges, S. Bridges, C. Sparrow, B. Wooley, B. Tang, and C. Jun. The development of an expert system for the characterization of containers of contaminated waste. Expert Systems with Applications, 17(3):167-181, 1999.

31. G. L. Kong, D. L. Xu, and J. B. Yang. Clinical decision support systems: a review of knowledge representation and inference under uncertainties. International Journal of Computational Intelligence System, 1(2):159-167, 2008.

32. G. Abowd, A. Bobick, I. Essa, E. Mynatt, and W. Rogers. The aware home: Developing technologies for successful aging. In Proceedings of AAAI Workshop on Automation as a Care Giver, pages 17. AAAI Press, July 2002.

33. H. Schaaf. Siemens' smart home (t-com house). Technical report, June 2005.

34. D. Kelly. Smart support at home: the integration of telecare technology with primary and community care systems. The British Journal of Healthcare Computing and Information Management, 22(3):19-21, 2005.

35. D. Cook and S. Das. Smart Environments: Technologies, Protocols and Applications. Wiley and Sons, 2005.

36. J. C. Augusto and C. D. Nugent. Designing Smart Homes: the role of Artificial Intelligence. Springer Verlag, 2006.

37. D. Cook. Health monitoring and assistance to support aging in place. Journal of Universal Computer Science - (Special Issue on "Pervasive Health Management: new challenges for Health Informatics”), 12(1), 2006.
38. Z. Z. Bien, H.-E. Lee, J.-H. Do, Y.-H. Kim, K.H. Park, and S.-E. Yang. Intelligent interaction for human-friendly service robot in smart house environment. International Journal of Computational Intelligence System, 1(1):77-93, 2008.

39. N. Paton and O. Diaz. Active database systems. ACM Computing Surveys, 31(1):63-103, 1999.

40. A. Galton. Qualitative Spatial Change. Oxford University Press, 2000.

41. R. Davis. Truth, Deduction, and Computation: Logic and Semantics for Computer Science. Computer Science Press, 1989.

42. R. Alur and T.A. Henzinger. Logics and Models of Real-Time: A Survey. In Real Time: Theory in Practice, volume 600, pages 74-106. Springer-Verlag, 1991.

43. G. Shafer. A Mathematical Theory of Evidence. Princeton University Press, Princeton, N.J., 1976.

44. C. L. Huang and K. Yoon. Multiple Attribute Decision Making Methods and Applications, A State-of-Art Survey. Springer-Verlag, 1981.

45. V. Belton and T. J. Stewart. Multiple Criteria Decision Analysis: An Integrated Approach. Kluwer, 2002.

46. L.A. Zadeh. Fuzzy sets. Information and Control, 8:338-353, 1965.

47. L. A. Zadeh. The concept of a linguistic variable and its application to approximate reasoning i. Information Science, 8:199-249, 1975.

48. L.A. Zadeh. The concept of a linguistic variable and its application to approximate reasoning ii. Information Science, 8:301-357, 1975.

49. L. A. Zadeh. The concept of a linguistic variable and its application to approximate reasoning iii. Information Science, 9:43-80, 1975.

50. J. B. Yang and M. G. Singh. An evidential reasoning approach for multiple attribute decision making with uncertainty. IEEE Transactions on Systems, Man, and Cybernetics, 24(1):1-18, 1994.

51. J. B. Yang and P. Sen. A general multi-level evaluation process for hybrid madm with uncertainty. IEEE Transactions on Systems, Man, and Cybernetics, 24(10):1458-1473, 1994.

52. J. B. Yang and D. L. Xu. Intelligent Decision System via Evidential Reasoning. IDSL, Cheshire, England, 1999.

53. J. B. Yang, J. Liu, D. L. Xu, J. Wang, and H. W. Wang. Optimisation models for training belief rule based systems. IEEE Transactions on Systems, Man, and Cybernetics, Part A: Systems and Humans, 37(4):569$585,2005$.

54. J. Liu, J.B. Yang, D. Ruan, L. Martinez, and J. Wang. Self-tuning of fuzzy belief rule bases for engineering system safety analysis. Annals of Operations Research, 163(1):143-168, 2008. 